UDK 517.5

\author{
V. F. Babenko*, Y. V. Babenko**, O. V. Kovalenko*** \\ * Oles Honchar Dnipro National University, \\ Dnipro 49050. E-mail: babenko.vladislav@gmail.com \\ ** Kennesaw State University, Marietta, GA 30060, USA, \\ Kyiv 03057. E-mail: ybabenko@kennesaw.edu \\ *** Oles Honchar Dnipro National University, \\ Dnipro 49050. E-mail: olegkovalenko90@gmail.com
}

\title{
On asymptotically optimal cubatures for multidimensional Sobolev spaces
}

\begin{abstract}
We find an asymptotically optimal method of recovery of the weighted integral for the classes of multivariate functions that are defined via restrictions on their (distributional) gradient.

Key words: Asymptotically optimal recovery methods, weighted cubature formulae, multidimensional Sobolev space.

Анотація. Знайдено асимптотично оптимальний метод відновлення інтеграла з вагою для класів функцій багатьох змінних, що задаються обмеженнями на (узагальнений) градієнт.

Ключові слова: Асимптотично оптимальні методи відновлення, вагові кубатурні формули, Соболевські класи функцій багатьох змінних.
\end{abstract}

MSC2020: Pri 41A55, SEC 41A44, 26D10

\section{Introduction}

Let $Q \subset \mathbb{R}^{d}, d \in \mathbb{N}$, be a nonempty, bounded, open set. By $W^{1, p}(Q)$, $p \in[1, \infty]$, we denote the Sobolev space of functions $f: Q \rightarrow \mathbb{R}$, such that $f$ and all their (distributional) first order partial derivatives belong to $L_{p}(Q)$. For $x=\left(x^{1}, \ldots, x^{d}\right) \in \mathbb{R}^{d}$ and $q \in[1, \infty)$, we set $|x|_{q}:=\left(\sum_{k=1}^{d}\left|x^{k}\right|^{q}\right)^{\frac{1}{q}},|x|_{\infty}:=$ $\max _{k=1, \ldots, d}\left|x^{k}\right|$. It is clear that for all $f \in W^{1, p}(Q)$ we have $\left\||\nabla f|_{1}\right\|_{L_{p}(Q)}<\infty$. For $p \in[1, \infty]$ set $W_{p}^{\nabla}(Q):=\left\{f \in W^{1, p}(Q):\left\||\nabla f|_{1}\right\|_{p} \leq 1\right\}$.

We say, that a set $Q \subset \mathbb{R}^{d}$ is composed of $m \in \mathbb{N}$ convex domains, if there exist disjoint convex domains $Q_{1}, \ldots, Q_{m}$ such that $Q=\bigcup_{k=1}^{m} Q_{k}$.

It is known (see e.g. $\S \S 1-3$ of Chapter 1 in [7]) that every bounded convex set is Jordan measurable, and hence a bounded set composed of a finite number of convex domains is also Jordan measurable. 
Everywhere in the article we consider only domains $Q$ composed of a finite number of convex domains. Such domains satisfy the so-called cone condition and hence for all $p>d$ each function from $W^{1, p}(Q)$ has a continuous representation, see e.g. Chapter 4 and Theorem 4.12 in [1].

Everywhere below we assume that $p>d \geq 2$. For $h>0$ we set

$$
\square_{h}^{d}:=\left\{x \in \mathbb{R}^{d}:|x|_{\infty} \leq h\right\} .
$$

For a finite set $A$, by $|A|$ we denote the number of its elements.

Let a measurable bounded set $Q \subset \mathbb{R}^{d}$, a class $X$ of continuous on $Q$ functions and $n \in \mathbb{N}$ be given. Let also $w$ be a non-negative integrable on $Q$ weight function. We consider the problem of optimal recovery of the integral $\int_{Q} w(x) f(x) d x$ of a function $f \in X$ based on $n$ function's values at points from $Q$. Arbitrary function $\Phi: \mathbb{R}^{n} \rightarrow \mathbb{R}$ is called a method of recovery. For given points $x_{1}, \ldots, x_{n} \in Q$ the error of recovery of the integral by the method $\Phi$ is defined by the equality

$$
e\left(X, \Phi, w, x_{1}, \ldots, x_{n}\right):=\sup _{f \in X}\left|\int_{Q} w(x) f(x) d x-\Phi\left(f\left(x_{1}\right), \ldots, f\left(x_{n}\right)\right)\right| .
$$

The problem of the optimal recovery of the integral is to find the best error of recovery

$$
E_{n}(X, w):=\inf _{x_{1}, \ldots, x_{n} \in Q} \inf _{\mathbb{R}^{n} \rightarrow \mathbb{R}} e\left(X, \Phi, w, x_{1}, \ldots, x_{n}\right)
$$

the best method of recovery and the best position of the informational set $x_{1}, \ldots, x_{n}$ (i.e. such method $\tilde{\Phi}: \mathbb{R}^{n} \rightarrow \mathbb{R}$ and points $\tilde{x_{1}}, \ldots, \tilde{x_{n}} \in Q$, for which the infima in (1) are attained), if such points and such a method exist. In the case when $w(x) \equiv 1$ on $Q$ we write $E_{n}(X)$ instead of $E_{n}(X, w)$.

In many cases it is hard to find the best error of recovery and optimal recovery method; in such situations it is interesting to find asymptotically optimal method of recovery, i.e. such sequence of methods $\Phi_{n}: \mathbb{R}^{n} \rightarrow \mathbb{R}$ and information sets $\left\{x_{1}^{n}, \ldots, x_{n}^{n}\right\}, n \in \mathbb{N}$, that

$$
\lim _{n \rightarrow \infty} \frac{E_{n}(X, w)}{e\left(X, \Phi_{n}, w, x_{1}^{n}, \ldots, x_{n}^{n}\right)}=1 .
$$

In the case $X=W_{p}^{\nabla}(Q)$ it is sufficient to consider only linear methods of recovery in (1). More precisely, the following lemma holds.

Lemma 1. For given points $x_{1}, \ldots, x_{n} \in Q$

$$
\inf _{\Phi: \mathbb{R}^{n} \rightarrow \mathbb{R}} \sup _{f \in W_{p}^{\nabla}(Q)}\left|\int_{Q} w(x) f(x) d x-\Phi\left(f\left(x_{1}\right), \ldots, f\left(x_{n}\right)\right)\right|
$$




$$
=\inf _{\substack{c_{k} \in \mathbb{R}, k=1, \ldots, n}} \sup _{f \in W_{p}^{\nabla}(Q)}\left|\int_{Q} w(x) f(x) d x-\sum_{k=1}^{n} c_{k} f\left(x_{k}\right)\right| .
$$

The existence of an optimal linear method of recovery is well known in many situations, in particular for convex centrally symmetric classes $X$. See for example Chapter $1 \S 3$ in [14].

Problems of optimal recovery are heavily studied and have a broad literature. We refer to the monographs $[10,14,13,12]$ for many results and further references.

This article is intimately connected with [4], where, in particular, the quantity $E_{n}\left(W_{p}^{\nabla}(Q)\right)$ for the case of convex bounded domain $Q$ was studied. The goal of this article is to find asymptotically optimal methods of the integral recovery with non-constant weight function.

Some results regarding optimal recovery of integrals with weights can be found in $[11,2,3,5,6]$

The article is organized as follows. In Section 2 we adduce necessary results from [4]. In Sections 3 and 4 we find the asymptotically optimal methods of the integral recovery for some classes of weight functions.

\section{Necessary known results}

\subsection{Extremal functions}

Let $p>d, p^{\prime}$ be such that $\frac{1}{p}+\frac{1}{p^{\prime}}=1$ and $h>0$. Consider the function $f_{e}: \square_{h}^{d} \rightarrow \mathbb{R}$

$$
f_{e}(y)=f_{e, h}(y)=\int_{0}^{|y|_{\infty}}\left|\frac{h^{d-1}}{u^{d-1}}-\frac{u}{h}\right|^{p^{\prime}-1} d u .
$$

In the proof of Theorem 3 in [4] it was shown, that $f_{e, h} \in W^{1, p}\left(\square_{h}^{d}\right)$ and

$$
\left|\nabla f_{e, h}(y)\right|_{1}=\left(\frac{h^{d-1}}{|y|_{\infty}^{d-1}}-\frac{|y|_{\infty}}{h}\right)^{p^{\prime}-1} \text { a.e. on } \square_{h}^{d} \text {. }
$$

Since $p\left(p^{\prime}-1\right)=p^{\prime}$,

$$
\left\|\left|\nabla f_{e, 1}\right|_{1}\right\|_{L_{p}\left(\square{ }_{1}^{d}\right)}^{p}=\left\|\frac{1}{|\cdot|_{\infty}^{d-1}}-|\cdot|_{\infty}\right\|_{L_{p^{\prime}}\left(\square_{1}^{d}\right)}^{p^{\prime}} .
$$

Set

$$
c(d, p):=\frac{1}{d}\left\|\frac{1}{|\cdot|_{\infty}^{d-1}}-|\cdot|_{\infty}\right\|_{L_{p^{\prime}}\left(\square_{1}^{d}\right)} .
$$


The restriction of the function $f_{e, h}$, to the boundary $\partial \square_{h}^{d}$ is constant; hence we can continuously continue the function $f_{e, h}$ to whole $\mathbb{R}^{d}$ by setting $f_{e, h}(y)$ equal to the value of $f_{e, h}$ on the boundary of $\square_{h}^{d}$ for all $y \notin \square_{h}^{d}$.

Let $Q \subset \mathbb{R}^{d}$ be a bounded region, $x_{1}, \ldots, x_{n} \in Q$ and $h>0$. For all $y \in \mathbb{R}^{d}$ set

$$
f_{h}\left(x_{1}, \ldots, x_{n} ; y\right):=\min _{k=1, \ldots, n} f_{e, h}\left(y-x_{k}\right) .
$$

It is easy to see that $f_{h}\left(x_{1}, \ldots, x_{n}\right) \in W^{1, p}(Q)$ for all $p \in(d, \infty]$.

For $x \in \mathbb{R}^{d}$ and $h>0$ set

$$
\square_{h}^{d}(x):=\left\{y \in \mathbb{R}^{d}:|x-y| \leq h\right\} .
$$

The following lemma is contained in [4], see Lemma 2.

Lemma 2. Let a bounded measurable domain $Q \subset \mathbb{R}^{d}, n \in \mathbb{N}$ and $h>0$ be given. If the points $\bar{x}_{1}, \ldots \bar{x}_{n} \in Q$ are such that the sets $\square_{h}^{d}\left(\bar{x}_{1}\right), \ldots, \square{ }_{h}^{d}\left(\bar{x}_{n}\right)$ are pairwise disjoint subsets of $Q$, then for all $x_{1}, x_{2}, \ldots, x_{n} \in Q$

$$
\int_{Q} f_{h}\left(x_{1}, \ldots, x_{n} ; y\right) d y \geq \int_{Q} f_{h}\left(\bar{x}_{1}, \ldots, \bar{x}_{n} ; y\right) d y \geq 0
$$

and for all $p \in(d, \infty]$

$$
\left\|\left|\nabla f_{h}\left(x_{1}, \ldots, x_{n}\right)\right|_{1}\right\|_{L_{p}(Q)} \leq\left\|\left|\nabla f_{h}\left(\bar{x}_{1}, \ldots, \bar{x}_{n}\right)\right|_{1}\right\|_{L_{p}(Q)} .
$$

\subsection{Asymptotically optimal information sets and weights}

In this section we recall the construction of the optimal information sets and weight for the problem of the integral optimal recovery in the case when $Q$ is Jordan measurable, see [4].

For $h>0$, we consider the lattice $\Lambda$ in $\mathbb{R}^{d}$ generated by the vectors $(2 h, 0,0, \ldots, 0),(0,2 h, 0,0, \ldots, 0), \ldots,(0, \ldots, 0,2 h) \in \mathbb{R}^{d}$. By $P_{k}(h)$ we denote the cubes

$$
2 h k+[0,2 h]^{d}, k \in \mathbb{Z}^{d} ;
$$

their volumes are equal to $(2 h)^{d}$. By $A(h)$, we denote the set of all cubes $P_{k}(h)$ that are contained in $Q$. Let $a(h)$ be the set of the centers of the cubes from $A(h)$. By $B(h)$ we denote the set of all cubes $P_{k}(h)$ that have non-empty set of common with $Q$ interior points. Let $b(h)$ be the set of the centers of the cubes from $B(h)$. Since $Q$ is Jordan measurable, we have $\lim _{h \rightarrow 0}|A(h)| \cdot(2 h)^{d}=$ $\lim _{h \rightarrow 0}|B(h)| \cdot(2 h)^{d}=\operatorname{mes} Q$ or, equivalently,

$$
|A(h)|=\frac{\operatorname{mes} \mathrm{Q}}{(2 h)^{d}}+o\left(\frac{1}{h^{d}}\right) \text { and }
$$




$$
|B(h)|=\frac{\operatorname{mes} \mathrm{Q}}{(2 h)^{d}}+o\left(\frac{1}{h^{d}}\right) \text { as } h \rightarrow 0 .
$$

Let $n \in \mathbb{N}$ be fixed. We set

$$
h_{n}:=\frac{1}{2}\left(\frac{\operatorname{mes} \mathrm{Q}}{n}\right)^{\frac{1}{d}}
$$

Then, due to (8),

$$
\left|A\left(h_{n}\right)\right|=n+o(n) \text { and }\left|B\left(h_{n}\right)\right|=n+o(n) \text { as } n \rightarrow \infty \text {. }
$$

For each cube $P$ from the set $B\left(3 h_{n}\right)$, we choose a point $z$ according to the following rule: $z$ is the center of the cube $P$ if it belongs to $a\left(h_{n}\right)$; otherwise, $z$ is an arbitrary point from $P \cap a\left(h_{n}\right)$ if the intersection is not empty, and $z$ is an arbitrary internal point of $Q \cap P$ if the intersection is empty.

By $S_{1}(n)$ we denote the set of such points $z$. From (8) it follows that the number $\left|S_{1}(n)\right|$ of points in $S_{1}(n)$ satisfies

$$
\left|S_{1}(n)\right|=\frac{n}{3^{d}}+o(n), \text { as } n \rightarrow \infty .
$$

Since for all $n \in \mathbb{N} a\left(3 h_{n}\right) \subset a\left(h_{n}\right)$, we obtain that

$$
\left|S_{1}(n) \backslash a\left(h_{n}\right)\right| \leq\left|B\left(3 h_{n}\right)\right|-\left|A\left(3 h_{n}\right)\right|=o(n) \text {, as } n \rightarrow \infty \text {. }
$$

Denote by $S_{2}(n)$ arbitrary subset of the set $a\left(h_{n}\right) \backslash S_{1}(n)$, that contains $n-$ $\left|S_{1}(n)\right|$ points (for large enough $n$ this number is positive; if $\left|a\left(h_{n}\right) \backslash S_{1}(n)\right| \leq$ $n-\left|S_{1}(n)\right|$, then we set $\left.S_{2}(n):=a\left(h_{n}\right) \backslash S_{1}(n)\right)$. Set $S(n):=S_{1}(n) \cup S_{2}(n)$.

Let $S(n)=\left\{x_{1}^{*}, \ldots, x_{|S(n)|}^{*}\right\}$. For each $k=1, \ldots,|S(n)|$, we define the set

$$
V_{k}=V_{k}(n):=\begin{aligned}
& \left\{x \in Q \cap P\left(3 h_{n} ; x_{k}^{*}\right):\left|x-x_{k}^{*}\right|_{\infty}<\left|x-x_{s}^{*}\right|_{\infty}, \quad s \neq k\right\},
\end{aligned}
$$

where $P\left(3 h_{n} ; x_{k}^{*}\right)$ is the cube from $B\left(3 h_{n}\right)$ that contains $x_{k}^{*}$. The sets $V_{k}$ are pairwise disjoint, $\bigcup_{k=1}^{|S(n)|} V_{k} \subset Q$, and mes $\left(Q \backslash \bigcup_{k=1}^{|S(n)|} V_{k}\right)=0$.

We set

$$
c_{k}^{*}:=\operatorname{mes} V_{k}, k=1, \ldots,|S(n)| \text {. }
$$

\subsection{Optimal recovery formulae}

An asymptotically optimal solution of the integral optimal recovery problem in the case of a convex domain and unit weight function is given by the following theorem, see [4]. 
Theorem 1. Let $d, n \in \mathbb{N}, p \in(d, \infty]$ and a bounded convex set $Q$ be given. Then

$$
E_{n}\left(W_{p}^{\nabla}(Q)\right)=c(d, p)\left(\frac{\operatorname{mes} Q}{2^{d}}\right)^{\frac{1}{d}+\frac{1}{p^{\prime}}} \cdot \frac{1+o(1)}{n^{\frac{1}{d}}}, n \rightarrow \infty,
$$

where the constant $c(d, p)$ is defined by (4). The asymptotically optimal information set is $S(n)$, defined in Section 2.2, and the optimal recovery method is

$$
\tilde{\Phi}_{n}\left(f\left(x_{1}\right), \ldots, f\left(x_{|S(n)|}\right)\right)=\sum_{k=1}^{|S(n)|} c_{k}^{*} f\left(x_{k}\right),
$$

where the weights $c_{k}^{*}$ are defined by $(14)$.

\section{Step weight functions}

Let $Q \subset \mathbb{R}^{d}$. We say that the weight function $w(x)$ is a step function, if there exists a number $m \in \mathbb{N}$ and disjoint sets $Q_{1}, \ldots, Q_{m}$ such that $Q=\bigcup_{k=1}^{m} Q_{k}$ and $w(x)=w_{k}>0$, for $x \in Q_{k}, k=1, \ldots, m$.

The goal of this section is to solve the problem of optimal integral recovery in the case, when the weight function is a step function and the sets $Q_{k}$ are convex.

\subsection{Optimal recovery with step weight function}

For each $j=1, \ldots, m$ and positive integer $n_{j}$ define the information set $S_{j}\left(n_{j}\right)=\left\{x_{k}^{j}\right\}_{k=1}^{\left|S_{j}\left(n_{j}\right)\right|}$ and weights $c_{k}^{j}, k=1, \ldots,\left|S_{j}\left(n_{j}\right)\right|$, the same way as it was done in Section 2.2, with the domain $Q$ substituted by $Q_{j}$ and the number $n$ by $n_{j}$.

The main result of this section is the following theorem.

Theorem 2. Let $p>d, w$ be a positive step function on the set $Q \subset \mathbb{R}^{d}$, i.e. $w(x)=w_{k}>0, x \in Q_{k}, k=1, \ldots, m$, where $Q=\bigcup_{k=1}^{m} Q_{k}$. Suppose that each of the sets $Q_{k}$ is convex. Then

$$
E_{n}\left(W_{p}^{\nabla}(Q), w\right)=\frac{c(d, p)(1+o(1))}{2^{1+\frac{d}{p^{\prime}}} n^{\frac{1}{d}}}\|w\|_{L_{q}(Q)}, n \rightarrow \infty,
$$

where $q=\frac{p^{\prime} d}{p^{\prime}+d}$ and $c(d, p)$ is defined by (4).

The asymptotically optimal information set is the set $S(n)=\bigcup_{j=1}^{m} S_{j}\left(n_{j}\right)$ and the asymptotically optimal recovery method is

$$
\tilde{\Phi}_{n}\left(f\left(x_{1}\right), \ldots, f\left(x_{|S(n)|}\right)\right)=\sum_{j=1}^{m} w_{j} \sum_{k=1}^{\left|S_{j}\left(n_{j}\right)\right|} c_{k}^{j} f\left(x_{k}^{j}\right),
$$


where the sets $S_{j}\left(n_{j}\right)$ and numbers $c_{k}^{j}$ are defined above,

$$
n_{j}=\left[|S(n)| \frac{w_{j}^{q} \operatorname{mes} Q_{j}}{\sum_{k=1}^{m} w_{k}^{q} \operatorname{mes} Q_{k}}\right], j=1, \ldots, m,
$$

and $[x]$ denotes the biggest integer not exceeding the real number $x$.

It is easy to see, that for an asymptotically optimal information set we have $n_{k} \rightarrow \infty$ as $n \rightarrow \infty$.

The proof of the theorem will be given in several subsections below.

\subsection{Auxiliary results}

We need the following lemma, which was used in several works, see e.g. [2]. We omit its technical proof.

Lemma 3. Let $m \in \mathbb{N}$, positive numbers $a_{1}, \ldots, a_{m}, n_{1}, \ldots, n_{m}, \alpha$ and $n$ such that $\sum_{k=1}^{m} n_{k}=n$ be given. Then

$$
\sum_{k=1}^{m} \frac{a_{k}}{n_{k}^{\alpha}} \geq \frac{1}{n^{\alpha}}\left(\sum_{k=1}^{m} a_{k}^{\frac{1}{\alpha+1}}\right)^{\alpha+1}
$$

The inequality turns into equality if for $k=1, \ldots, m$

$$
n_{k}=n \frac{a_{k}^{\frac{1}{\alpha+1}}}{\sum_{k=1}^{m} a_{k}^{\frac{1}{\alpha+1}}}
$$

Lemma 4. Let a Jordan measurable set $Q \subset \mathbb{R}^{d}$ be given. For a given $h>0$ consider the set

$$
Q^{h}:=\left\{x \in Q: \inf _{y \in \partial Q}|x-y|_{\infty} \geq h\right\}
$$

Then mes $Q^{h}=\operatorname{mes} Q+o(1), h \rightarrow 0$.

Proof. Using notations from Section 2.2 and equalities (8) we have $|B(h)\rangle$ $A(h) \mid=o\left(h^{-d}\right), h \rightarrow 0$. For each cube $P \in B(h) \backslash A(h)$ (with length of the edge equal to $2 h$ ) consider the cube $\tilde{P}$ with the same center and the length of the edge equal to $4 h$. Set $\tilde{Q}:=Q \backslash \bigcup_{P \in B(h) \backslash A(h)} \tilde{P}$. Then $Q^{h} \supset \tilde{Q}$ and $\operatorname{mes} \tilde{Q} \geq \operatorname{mes} Q-o\left(h^{-d}\right) \cdot(4 h)^{d}=\operatorname{mes} Q+o(1), h \rightarrow 0$. The lemma is proved. 


\subsection{Estimate from above}

For each set of quantities $n_{k}$ of information nodes in the sets $Q_{k}, k=$ $1, \ldots, m$, choose the information nodes $x_{k}^{i}$ and coefficients $c_{k}^{i}, i=1, \ldots, n_{k}$, to be optimal for each of the set $Q_{k}$. Using Lemma 1, Theorem 1, Holder's inequality and Lemma 3 we obtain (as $n \rightarrow \infty$ )

$$
\begin{gathered}
E_{n}\left(W_{p}^{\nabla}(Q), w\right) \leq \inf _{n_{i}} \sup _{f \in W_{p}^{\nabla}(Q)} \sum_{i=1}^{m} w_{i}\left|\int_{Q_{i}} f(x) d x-\sum_{k=1}^{n_{i}} c_{k}^{i} f\left(x_{k}^{i}\right)\right| \\
\leq \inf _{n_{i}} \sup _{f \in W_{p}^{\nabla}(Q)} \sum_{i=1}^{m} w_{i}\left\||\nabla f|_{1}\right\|_{L_{p}\left(Q_{i}\right)} \sup _{g \in W_{p}^{\nabla}\left(Q_{i}\right)}\left|\int_{Q_{i}} g(x) d x-\sum_{k=1}^{n_{i}} c_{k}^{i} g\left(x_{k}^{i}\right)\right| \\
=\inf _{n_{i}} \sup _{f \in W_{p}^{\nabla}(Q)} \sum_{i=1}^{m} w_{i}\left\||\nabla f|_{1}\right\|_{L_{p}\left(Q_{i}\right)} E_{n_{i}}\left(W_{p}^{\nabla}\left(Q_{i}\right)\right) \\
\text { (by Theorem 1) }
\end{gathered}
$$$$
=\inf _{n_{i}} \sup _{f \in W_{p}^{\nabla}(Q)} \sum_{i=1}^{m} w_{i} c(d, p)\left(\frac{\operatorname{mes} Q_{i}}{2^{d}}\right)^{\frac{1}{d}+\frac{1}{p^{\prime}}} \frac{1+o(1)}{n_{i}^{\frac{1}{d}}}\left\||\nabla f|_{1}\right\|_{L_{p}\left(Q_{i}\right)}
$$

(applying Holder's inequality)

$$
\begin{array}{r}
\leq c(d, p)(1+o(1)) \inf _{n_{i}} \sup _{f \in W_{p}^{\nabla}(Q)}\left(\sum_{i=1}^{m} \frac{w_{i}^{p^{\prime}}}{n_{i}^{\frac{p^{\prime}}{d}}}\left(\frac{\operatorname{mes} Q_{i}}{2^{d}}\right)^{\frac{p^{\prime}}{d}+1}\right)^{\frac{1}{p^{\prime}}} \\
\cdot\left(\sum_{i=1}^{m}\left\||\nabla f|_{1}\right\|_{L_{p}\left(Q_{i}\right)}^{p}\right)^{\frac{1}{p}}
\end{array}
$$

(using Lemma 3)

$$
=\frac{c(d, p)(1+o(1))}{n^{\frac{1}{d}}}\left(\sum_{i=1}^{m} w_{i}^{\frac{p^{\prime}}{1+\frac{p^{\prime}}{d}}} \frac{\operatorname{mes} Q_{i}}{2^{d}}\right)^{\frac{1+\frac{p^{\prime}}{d}}{p^{\prime}}}=\frac{c(d, p)(1+o(1))}{2^{1+\frac{d}{p^{\prime}}} n^{\frac{1}{d}}}\|w\|_{L_{q}(Q)} .
$$

\subsection{Glued extremal functions}

For each configuration of the information set $x_{1}, \ldots, x_{n}$, and weights $w_{1}, \ldots, w_{n}$, we define a function $f: Q \rightarrow \mathbb{R}$ by the following construction. For each $i=1, \ldots, m$, define a function $f_{i}(x): Q_{i} \rightarrow \mathbb{R}$ by the formula

$$
f_{i}(x):=f_{h_{i}}\left(x_{1}^{i}, \ldots, x_{n_{i}}^{i} ; x\right),
$$

where, the points $x_{1}^{i}, \ldots, x_{n_{i}}^{i}$ are the points from information set that belong to $Q_{i}, h_{i}:=\frac{1}{2}\left(\frac{\operatorname{mes} Q_{i}}{n_{i}}\right)^{\frac{1}{d}}$ and the functions $f_{h}$ are defined by $(5)$. 
Set

$$
g(x):=\min _{y \in \bigcup_{k=1}^{m} \partial Q_{k}}|x-y|
$$

and

$$
f(x)=w_{i}^{\frac{d}{p d+p-d}} \min \left\{g(x), f_{i}(x)\right\}, x \in Q_{i}, i=1, \ldots, m .
$$

Due to the triangle inequality,

$$
|g(x)-g(y)| \leq|x-y|
$$

for all $x, y \in Q$, hence the function $g$ is Lipschitz. Thus, due to the Rademacher theorem, there exists (classical) gradient of $g$ almost everywhere. Moreover, it agrees with the distributional gradient almost everywhere. Hence, due to (16), $g \in W^{1, \infty}(Q)$, see for example [8], Sections 3 and 4. Therefore $f \in W^{1, p}(Q)$ and $\frac{f}{\left\||\nabla f|_{1}\right\|_{L_{p}(Q)}} \in W_{p}^{\nabla}(Q)$.

Note, that $f \equiv w_{i}^{\frac{d}{p d+p-d}} f_{h_{i}}\left(x_{1}^{i}, \ldots, x_{n_{i}}^{i}\right)$ on $Q_{i}^{H_{i}}$, where $H_{i} \rightarrow 0$ as $h_{i} \rightarrow 0$ and the notation $Q^{h}$ is defined in Lemma 4 .

Denote by $\tilde{f}$ the function $f$ with $x_{1}, \ldots, x_{n}$ chosen in such a way, that for all $i=1, \ldots, m$, the subsets $x_{1}^{i}, \ldots, x_{n_{i}}^{i}$ are optimal information sets in Theorem 1 (with $Q$ substituted by $Q_{i}$ and $n$ by $n_{i}$ ). Set $\tilde{Q}=\tilde{Q}\left(h_{1}, \ldots, h_{m}\right):=\bigcup_{i=1}^{m} Q_{i}^{H_{i}}$. By Lemma 4 , mes $\tilde{Q}=\operatorname{mes} Q+o(1), n \rightarrow \infty$.

We need the following lemma.

Lemma 5. For each $i=1, \ldots, m$ as $n \rightarrow \infty$

$$
\left\||\nabla \tilde{f}|_{1}\right\|_{L_{p}\left(Q_{i}^{H_{i}}\right)}^{p}=(1+o(1)) w_{i}^{\frac{d p}{d p+p-d}} \frac{\operatorname{mes} Q_{i}^{H_{i}}}{2^{d}} \int_{\square_{1}^{d}}\left(\frac{1}{|x|_{\infty}^{d-1}}-|x|_{\infty}\right)^{p^{\prime}} d x .
$$

Proof. By the definition of the function $\tilde{f}$ and Lemma 4 we have

$$
\left\||\nabla \tilde{f}|_{1}\right\|_{L_{p}\left(Q_{i}^{H_{i}}\right)}^{p}=(1+o(1)) n_{i} w_{i}^{\frac{d p}{d p+p-d}}\left\|\left|\nabla f_{e, h_{i}}\right|_{1}\right\|_{L_{p}\left(\square_{h_{i}}^{d}\right)}^{p} .
$$

Since $\left(p^{\prime}-1\right) p=p^{\prime}$, from (3) we obtain

$$
\begin{gathered}
\left\|\left|\nabla f_{e, h_{i}}\right|_{1}\right\|_{L_{p}\left(\square_{h_{i}}^{d}\right)}^{p}=\int_{\square_{h_{i}}^{d}}\left(\frac{h_{i}^{d-1}}{|x|_{\infty}^{d-1}}-\frac{|x|_{\infty}}{h_{i}}\right)^{p^{\prime}} d x \\
=h_{i}^{d} \int_{\square_{1}^{d}}\left(\frac{1}{|y|_{\infty}^{d-1}}-|y|_{\infty}\right)^{p^{\prime}} d y .
\end{gathered}
$$

To finish the proof of the lemma it is sufficient to notice that $n_{i} h_{i}^{d}=\frac{\operatorname{mes} Q_{i}}{2^{d}}$. The lemma is proved. 


\subsection{Estimate from below}

For each information set $x_{1}, \ldots, x_{n}$ and weights $c_{1}, \ldots, c_{n}$,

$$
\begin{gathered}
\sup _{g \in W_{p}^{\nabla}(Q)}\left|\int_{Q} w(x) g(x) d x-\sum_{k=1}^{n} c_{k} g\left(x_{k}\right)\right| \geq \frac{1}{\left\||\nabla f|_{1}\right\|_{L_{p}(Q)}} \int_{Q} w(x) f(x) d x \\
\left(\text { by Lemma 2) } \geq \frac{1}{\left\||\nabla \tilde{f}|_{1}\right\|_{L_{p}(Q)}} \sum_{i=1}^{m} w_{i} \int_{Q_{i}} \tilde{f}_{i}(x) d x\right. \\
\text { (by Lemma 4) }=\frac{1+o(1)}{\left\||\nabla \tilde{f}|_{1}\right\|_{L_{p}(\tilde{Q})}} \sum_{i=1}^{m} w_{i} \int_{Q_{i}^{H_{i}}} \tilde{f}_{i}(x) d x
\end{gathered}
$$

(by extremality of the function $\tilde{f}$ in Theorem 1)

$$
=\frac{1+o(1)}{\left\||\nabla \tilde{f}|_{1}\right\|_{L_{p}(\tilde{Q})}} \sum_{i=1}^{m} w_{i} c(d, p)\left(\frac{\operatorname{mes} Q_{i}^{H_{i}}}{2^{d}}\right)^{\frac{1}{d}+\frac{1}{p^{\prime}}}\left\||\nabla \tilde{f}|_{1}\right\|_{L_{p}\left(Q_{i}^{H_{i}}\right)} n_{i}^{-\frac{1}{d}}
$$

(by Lemma 5)

$$
\begin{gathered}
=\frac{c(d, p)(1+o(1))}{\left(\sum_{i=1}^{m} w_{i}^{\frac{p d}{p d+p-d}} \frac{\operatorname{mes} Q_{i}^{H_{i}}}{2^{d}}\right)^{\frac{1}{p}} \sum_{i=1}^{m} w_{i}^{\frac{p d+p}{p d+p-d}}\left(\frac{\operatorname{mes} Q_{i}^{H_{i}}}{2^{d}}\right)^{\frac{1}{d}+\frac{1}{p^{\prime}}}\left(\frac{\operatorname{mes} Q_{i}^{H_{i}}}{2^{d}}\right)^{\frac{1}{p}} n_{i}^{-\frac{1}{d}}} \\
=\frac{c(d, p)(1+o(1))}{\left(\sum_{i=1}^{m} w_{i}^{\frac{p d}{p d+p-d}} \frac{\operatorname{mes} Q_{i}^{H_{i}}}{2^{d}}\right)^{\frac{1}{p}}} \sum_{i=1}^{m} w_{i}^{\frac{p d+p}{p d+p-d}}\left(\frac{\operatorname{mes} Q_{i}^{H_{i}}}{2^{d}}\right)^{\frac{1}{d}+1} n_{i}^{-\frac{1}{d}}
\end{gathered}
$$

(by Lemma 3$) \geq \frac{c(d, p)(1+o(1))}{n^{\frac{1}{d}}\left(\sum_{i=1}^{m} w_{i}^{\frac{p d}{p d+p-d}} \frac{\operatorname{mes} Q_{i}^{H_{i}}}{2^{d}}\right)^{\frac{1}{p}}}\left(\sum_{i=1}^{m} w_{i}^{\frac{p d}{p d+p-d}} \frac{\operatorname{mes} Q_{i}^{H_{i}}}{2^{d}}\right)^{\frac{d+1}{d}}$

$$
\begin{gathered}
=\frac{c(d, p)(1+o(1))}{n^{\frac{1}{d}}}\left(\sum_{i=1}^{m} w_{i}^{\frac{p d}{p d+p-d}} \frac{\operatorname{mes} Q_{i}^{H_{i}}}{2^{d}}\right)^{\frac{p d+p-d}{p d}} \\
=\frac{c(d, p)(1+o(1))}{2^{1+\frac{d}{p^{\prime}}} n^{\frac{1}{d}}}\|w\|_{L_{q}(Q) \cdot}
\end{gathered}
$$

\section{Main results}

The goal of this section is to solve the problem of asymptotically optimal integral recovery in the case when the weight function is admissible. 


\subsection{Definition and some examples}

Let a bounded set $Q \subset \mathbb{R}^{d}$ be given. We call a function $w: Q \rightarrow \mathbb{R}$ admissible if it is positive almost everywhere, bounded, and there exists $M \in \mathbb{N}$, such that for all $C_{1}<C_{2}$ the set $\left\{x \in Q: w(x) \in\left[C_{1}, C_{2}\right]\right\}$ is composed of $m \leq M$ convex domains.

First of all note, that the step functions are admissible, if the sets $Q_{k}$ are composed of convex domains. Another set of admissible functions can be constructed as follows.

Let $\varphi:[0,1] \rightarrow \mathbb{R}$ be a monotone function. Consider the function $w=$ $w(\varphi): \square_{1}^{d} \rightarrow \mathbb{R}$, defined by the formula $w(x)=\varphi\left(|x|_{\infty}\right)$. For arbitrary $C_{1}<$ $C_{2}$, the set $\varphi^{-1}\left[C_{1}, C_{2}\right]$ is equal to $[\alpha, \beta] \backslash X$, where $0 \leq \alpha \leq \beta \leq 1$ and $X \subset\{\alpha, \beta\}$. Hence the set $w^{-1}\left[C_{1}, C_{2}\right]$ is equal to $\square_{\beta}^{d} \backslash \square_{\alpha}^{d}$ (with appropriately included boundaries). The latter set is a union of $2 d$ convex sets. Indeed, let for definiteness $X=\emptyset$. Then the convex sets can be chosen as

$$
T_{1}^{ \pm}=\square_{\beta}^{d} \cap\left\{ \pm x_{1} \in[\alpha, \beta]\right\},
$$

and for $k=2, \ldots, d$

$$
T_{k}^{ \pm}=\square_{\beta}^{d} \cap\left\{ \pm x_{k} \in[\alpha, \beta],\left|x_{1}\right|, \ldots,\left|x_{k-1}\right|<\alpha\right\} .
$$

hence the weight $w$ is admissible and $M$ from the definition can be set equal to $2 d$.

Using the arguments above, one can prove that the function $w(\varphi)$ remains admissible, if one or several assumptions are modified:

1. Request $\varphi$ to be only a piecewise-monotone function; i.e. such that there exists a partition $0=t_{0}<t_{1}<\ldots<t_{n}=1$, so that $\varphi$ is monotone on each of the intervals $\left(t_{i-1}, t_{i}\right), i=1, \ldots, n$.

2. Substitute the set $\square_{1}^{d}$ by an arbitrary convex domain $Q$.

3. Substitute the norm $|\cdot|_{\infty}$ by the norm $|\cdot|_{1}$.

\subsection{Solution of the integral optimal recovery problem}

Theorem 3. Let $p>d, w$ be admissible function on $Q \subset \mathbb{R}^{d}$. Then

$$
E_{n}\left(W_{p}^{\nabla}(Q), w\right)=\frac{c(d, p)(1+o(1))}{2^{1+\frac{d}{p^{\prime}}} n^{\frac{1}{d}}}\|w\|_{L_{q}(Q)}, n \rightarrow \infty
$$

where $q=\frac{p^{\prime} d}{p^{\prime}+d}$ and $c(d, p)$ is defined by (4).

Proof. Note, that if for all $x \in Q$ we have $w(x) \leq v(x)$, then

$$
E_{n}\left(W_{p}^{\nabla}(Q), w\right) \leq E_{n}\left(W_{p}^{\nabla}(Q), v\right) .
$$


This inequality follows from the existence of an optimal linear method of recovery (see Lemma 1 ) and the equality

$$
\begin{array}{r}
\inf _{\substack{c_{k} \in \mathbb{R}, k=1, \ldots, n}} \sup _{f \in W_{p}^{\nabla}(Q)}\left|\int_{Q} w(x) f(x) d x-\sum_{k=1}^{n} c_{k} f\left(x_{k}\right)\right| \\
=\sup _{\substack{f \in W_{p}^{\nabla}(Q), f\left(x_{k}\right)=0, k=1, \ldots, n}} \int_{Q} w(x) f(x) d x,
\end{array}
$$

which can be proved using the standard arguments involving the Hahn-Banach theorem, see e.g. [9, Theorem 1.3.4].

Let $c \leq w(x) \leq C$ for all $x \in Q$. Fix $m \in \mathbb{N}$, and for $k=1, \ldots, m$ set

$$
Q_{k, m}:=\left\{x \in Q: w(x) \in\left[c+(k-1) \frac{C-c}{m}, c+k \frac{C-c}{m}\right]\right\} .
$$

Consider the function $\bar{w}_{m}(x)=c+k \frac{C-c}{m}$ for all $x \in Q_{k, m}, k=1, \ldots, m$. Then $w(x) \leq \bar{w}_{m}(x)$ for all $x \in Q$ and using Theorem 2 we obtain

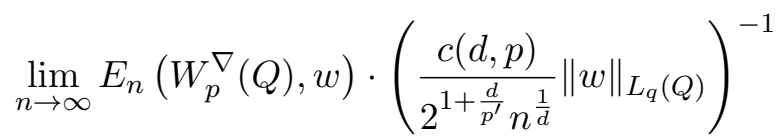

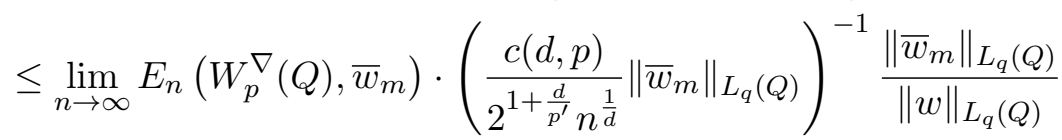

$$
\begin{aligned}
& =\frac{\left\|\bar{w}_{m}\right\|_{L_{q}(Q)}}{\|w\|_{L_{q}(Q)}}
\end{aligned}
$$

Since $\left\|\bar{w}_{m}\right\|_{L_{q}(Q)} \rightarrow\|w\|_{L_{q}(Q)}$ as $m \rightarrow \infty$ we obtain

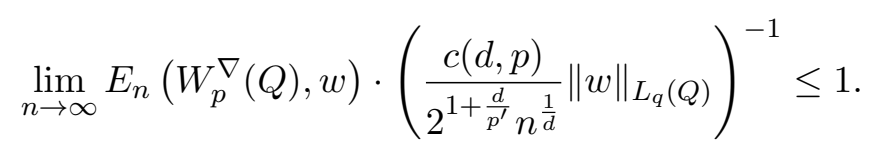

Applying the same arguments to the function $\underline{w}_{m}(x)=c+(k-1) \frac{C-c}{m}$ for all $x \in Q_{k, m}, k=1, \ldots, m$, we can get the inequality

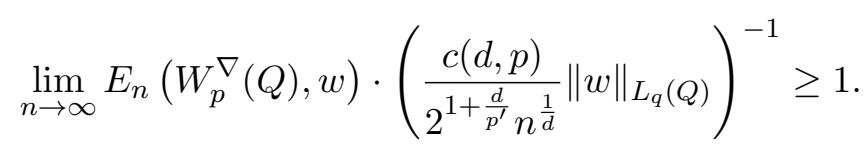

The theorem is proved.

\section{References}

1. Adams R.A., Fournier J.J.F.: Sobolev Spaces, ISSN. Elsevier Science, 2003. 
2. Babenko V.F.: Faithful asymptotics of remainders optimal for some classes of functions with cubic weight formulas. Math. Notes 20(4) (1976), 887-890.

3. Babenko V.F.: On optimization of weight quadrature formulas. Ukrainian Math. J. $47(8)$ (1995), 1157-1168.

4. Babenko V.F., Babenko Yu.V., Kovalenko O.V.: On multivariate Ostrowski type inequalities and their applications. Math. Ineq. Appl. 23(2) (2020), 569-583.

5. Chernaya E.V.: Asymptotically exact estimation of the error of weighted cubature formulas optimal in some classes of continuous functions. Ukrainian Math. J. 47(10) (1995), 1606-1618.

6. Chernaya E.V.: On the optimization of weighted cubature formulae on certain classes of continuous functions. East J. Approx. 1 (1995), 47-60.

7. Gruber P.M., Lekkerkerker C.G.: Geometry of Numbers. Bibliotheca mathematica. North-Holland, 1987.

8. Heinonen J.: Lectures on Lipschitz Analysis. Bericht (Jyväskylän yliopisto. Matematiikan ja tilastotieteen laitos). University of Jyväskylä, 2005.

9. Korneichuk N.: Exact Constants in Approximation Theory. Encyclopedia of Mathematics and its Applications. Cambridge University Press, 1991.

10. Nikolsky S.M.: Quadrature Formulae. Nauka, Moscow, 1979.

11. Polovinkin V.I.: Some estimates of norms of functionals of cubature formula errors. Math. Notes 5(3) (1969), 192-195.

12. Sobolev S.L., Vaskevich V.L.: The Theory of Cubature Formulas. Mathematics and Its Applications. Springer Netherlands, 2013.

13. Traub J.F., Wozhniakovski H.: A general theory of optimal algorithms. Acad. Press, 1980.

14. Zhensykbaev A.A.: Problems of recovery of operators. Moscow-Izhevsk, 2003.

Received: 19.11.2021. Accepted: 24.12.2021 